\title{
Pevista
}

ISSN-L: 2215-4728

DOI: http://dx.doi.org/10.15359/rp.23.4

http://www.revistas.una.ac.cr/perspectivas

N. ${ }^{\circ}$ 23. Julio-Diciembre, $2021 \cdot$ pp. 1-22

\section{Perspectivas, cambios y resignificación de la enseñanza de la historia en Honduras del Bicentenario}

\author{
Perspectives, Changes and Resignification of \\ the Teaching of History in the Honduras of the \\ Bicentennial
}

\author{
Erick José Guevara Pineda*
}

Fecha de recepción: 13/03/2021 - Fecha de aceptación: 07/05/2021

Resumen: las nuevas dinámicas sociales han provocado un giro significativo en la enseñanza de las ciencias sociales y particularmente, en la historia, este artículo tiene como fin la presentación de un estudio de caso descriptivo no experimental, desarrollado en el centro asociado Choluteca de la Universidad Metropolitana de Honduras -ubicado en el municipio de Choluteca- y el Instituto Oficial Villa Marco -ubicado en el municipio de Marcovia- en la región sur de Honduras. Los cuales han permitido la señalización de elementos a deconstruir para la comprensión de los procesos que como sociedades bicentenarias debemos consolidar o bien resignificar. Este trabajo trata la
Abstract: The new social dynamics have caused a significant turn in the teaching of the social sciences and particularly in history. The purpose of this article is to present a non-experimental descriptive case study; developed at the Choluteca associated center of the Metropolitan University of Honduras -located in the municipality of Choluteca- and the Villa Marco Official Institute -located in the municipality of Marcovia- in the southern region of Honduras. Which have allowed the marking of elements to be deconstructed for the understanding of the processes that as bicentennial societies we must consolidate or re-signify. This work deals with the need to merge the history

* Hondureño. Licenciado en Historia por la Universidad Nacional Autónoma de Honduras (UNAH), Ciudad Universitaria, Tegucigalpa, Honduras. Docente e investigador en la Universidad Metropolitana de Honduras (UMH), Centro Asociado Choluteca, Choluteca, Honduras. Docente en el Instituto Oficial "Villa Marco" (IVM) Marcovia, Honduras. Correo electrónico: erick.guevara@umh.edu.hn ORCID: https://orcid.org/0000-0001-8990-3537 
necesidad de fusionar la historia enseñada de la investigada, acoplando una nueva visión de cómo abordar los contenidos curriculares, el fortalecimiento de la toma de decisión po parte de los estudiantes, y algunas consideraciones que como docentes en la interacción virtual o semipresencial debemos hacer valer en la Honduras del siglo XXI, como la debida dosificación de los contenidos, e manejo de las plataformas o las aulas virtuales, así como de juegos en tiempo real que permitan la idea de interacción constante el seguimiento a los estudiantes que lo ameriten con mayor necesidad .

Palabras claves: Estudios Sociales; Enseñanza de la Historia; educación en pandemia; historia; Bicentenario, Honduras.

Tras los acontecimientos sucedidos durante el año 2020, el mundo pasa por una especie de bucle, donde las sociedades se ven sometidas a una pausa obligada, a ella se le suma una necesidad de reflexionar sobre lo que hasta ese momento era considerado una especie de "normalidad" discursos que se verán robustecidos por los gobiernos de las diferentes naciones al adoptar medidas que permitieran la contención de los casos

Sí bien en cierto que la pandemia por coronavirus fue tremendamente significativa en el nivel mundial, desde años atrás todos los sectores venían desarrollando una serie de cambios acelerados por una sociedad altamente fluctuante y exigente, añadiendo que en esta sociedad lo único permanente es el cambio. Bajo esta concepción social es que se puede orientar al mundo de la enseñanza de las ciencias sociales en general y la historia en particular, embarcándonos en un estudio de caso descriptivo no experimental por la naturaleza misma del análisis.

\section{La crisis y la reconstrucción de las ciencias sociales}

Debemos considerar que la función educadora estrictamente hablando consiste en la difusión de los saberes en diferentes áreas, en cambio la creación de los conocimientos no es otra cosa que los procesos de investigación relativos a instituciones, gobiernos y personas independientes que buscan profundizar en determinadas temáticas; sabiendo esto, si bien ambas pareciesen ser diferentes están complementadas en una especie de función, que podríamos llamar, generación de ciencia o historia como ciencia.

Esta generación del conocimiento en el área que nos compete a sido profundamente discriminada a través de los años, el estandarte de todo desmérito a las ciencias sociales siempre será la subjetividad o parcialidad. Paradójicamente, el mundo previo a la pandemia se llenó mucho de las llamadas pseudociencias haciéndonos recordar la Academia de Lagado, donde uno de sus investigadores quería obtener rayos de sol a partir de pepinos, en tanto otro buscaba reducir el excremento humano al alimento que le había dado origen dividiendo las partículas, liberándolo del color y olor (Bunge, 2002, pág. 212), en fin, esta posmodernidad cerco a muchos educadores a que en sus clases existieran muchos contenidos interesantes, pero poco importantes. Aún más en los contextos regionales de Centroamérica.

En contraposición a esta óptica la misma historia ha dado giros buscando resignificación de los procesos, la obra de Jo Guldi junto a David Armitage, pretende retornar a los estudios de larga duración, evitar a toda costa ese cortoplacismo placentero, pero dañino (Guldi \& Armitage, 2016), esto en virtud de la necesidad de comprender la realidad no como una especie de designación divina, sino como resultado de toda una historia.

Es importante añadir que previo a la pandemia algunos centros de educación superior se habían adelantado a la renovación de los profesionales de la enseñanza y la producción de la historia, ejemplo de ello, el importante estudio realizado por Paula Pantoja quien en su teoría fundamentada (Pantoja Suárez, 2017), busca hacer ver a los cursante de la carrera docente en el área de ciencias sociales la necesidad inminente de integrar realidades a fin de construir en los educandos la conciencia

Perspectivas, cambios y resignificación de la enseñanza de la historia en Honduras del Bicentenario 
histórica. Esta postura es simbólica si comprendemos que solo a través de la historia, es decir solo a través de la enseñanza de un recorrido cultural de larga duración es como se logra construir identidad.

En el caso particular de la historia como ciencia los procesos de reconstrucción permiten una constante interpretación de la enseñanza misma, tal como lo explica Joaquín Prats al señalar que la historia misma se ve ligada a una dialéctica de los hechos, al no poseer tubos o laboratorios de ensayos en los cuales las muestras sean enteramente aceptadas y generalizada (Prats, 2001), o bien esa historia enseñada que da legitimidad a ciertos discursos oficialistas de diferentes gobiernos.

A modo que esa constante producción y difusión logra justamente que la historia, aunque se vea sumergida en diferentes ópticas, posturas o escuelas nunca llegue a su fin, como se aseveró con una intencionalidad determinada hacia finales del siglo pasado (Fujuyama, s.f.). Sintetizaré este primer acercamiento epistemológico, en que la historia siempre tendrá una funcionalidad para las sociedades, la comprensión de acontecimientos desarrollados en el tiempo histórico, las causas de las problemáticas o las realidades, o las investigaciones que permiten esa relativización de las situaciones -comparar pasado, presente- (Prats, 2001, pág. 69), siempre existirá una necesidad de aprender historia, pero sobre todo de aprender a hacer historia.

\section{El nuevo imaginario de la enseñanza, conviviendo con SARS-CoV-2}

Si bien es cierto que algunos países de la región centroamericana tenían las experiencias de los escenarios virtuales (Parrales, 2020), la mayoría intentaba a soplos adaptar alguna herramienta informática a las clases, desde la experiencia en el nivel medio y superior los docentes que intentaban modernizar los espacios pedagógicos fueron discriminados en el caso de Honduras, haciendo valer siempre la longevidad del docente sobre la innovación de algunos de los nuevos profesionales.

Ese marco da un claro contexto de lo poco o casi nada preparados que se encontraba en la cintura de América para el salto de lo presencial a lo digital. Lo que a continuación mostraré es producto de una metodología de estudio de caso- Honduras- y un universo de muestra -los estudiantes de la universidad Metropolitana de Honduras, Centro Asociado Choluteca, ubicado en el municipio de Choluteca y del Instituto Oficial "Villa Marco", ubicado en el municipio de Marcovia- al ser este estudio no experimental, sino que descriptivo. Dejando claro además que se refiere a la enseñanza de la historia como asignatura obligatoria en los planes académicos y no una visión de la carrera de Historia.

La revolución informática ha sido toda una evolución social, político, económica que va desde posterior de la Segunda Guerra Mundial, con la utilización de codificadores y decodificadores (Bejerano, 2014), hasta nuestra actualidad con la llamada web 4.0; en materia educativa los avances gracias a esta evolución ha significado la posibilidad de aumentar los potenciales académicos que van desde una videoclase, hasta la consulta de repositorios digitales, herramientas didácticas y juegos interactivos, una serie de posibilidades que fácilmente haría de una clase de historia, sociología, ética profesional, psicología, economía y fundamentos de la investigación una experiencia para los estudiantes exquisita.

Ahora bien esta realidad es ajena al caso hondureños donde el Currí culum Nacional Básico máximo orientador de los pénsum académicos de las clases en los niveles prebásicos, básicos y medios, proyectaba nada más la utilización de videos disponibles en la web, carteles con imágenes, murales y lluvias de ideas -técnicas que son útiles, pero están desactualizadas- de igual manera en el ámbito de las universidades la utilización de un proyector representó la ambigüedad de lo que se conoce como gamificación pedagógica por parte de los llamados "catedráticos".

La inestabilidad profunda de las instituciones del Estado, los constantes señalamientos de secuestro por crimen organizado y narcotráfico, genera que los estudiantes se cuestionen si realmente, vale la pena estudiar, si es más factible migrar o bien buscar un trabajo para sobrevivir. Por lo tanto, la enseñanza de las ciencias sociales y específicamente, la historia en el caso de Honduras hace que cobre una necesidad inherente para la recuperación de la dignidad de los ciudadanos honuderños.

Marzo del año 2020 marca por sí mismo un antes y un después en la educación del país, en el sector oficial sustentado por los docentes, los padres de familia y los estudiantes se da paso a una embrionaria idea de virtualización de los espacios pedagógicos, en cambio las universidades en tiempo récord logran la virtualización de todos los campus que 
la mayoría ya poseían (Diario La Prensa, 2020). Este fenómeno es sin duda un punto que da tanta información hasta para la redacción de una monografía o tesis, sin embargo, por situaciones de espacio, así como la naturaleza misma del escrito lo resumiré en dos apartados.

\subsection{La virtualización de las ciencias sociales en el nivel superior en Honduras}

Una de las primeras decisiones por parte del Consejo Superior Universitario en Honduras fue el cierre físico de los campus de todas las universidades, (UNAH, 2020) medida acatada por todos los centros de estudio superior, incluyendo la Universidad Metropolitana de Honduras que forma parte de nuestra muestra.

A ello, le siguió casi de manera inmediata una serie de capacitaciones intensivas con duraciones de hasta ocho horas seguidas con el fin de acoplar al docente con las mediación digital -aulas virtuales y aplicaciones de clases en tiempo real- todo ello representó un esfuerzo considerable, pero muy fructífero; los colegas de las áreas de sociales por ejemplo, destinaban parte de su tiempo para practicar entre ellos dichas herramientas. Este giro educativo representó un cambio de paradigma en la forma de dar clases, señalaba Michael Parrales "Pasamos de tener respuestas a las preguntas, a hacer nosotros las preguntas" refiriéndose a los docentes (Parrales, 2020).

Los hondureños en el nivel superior materializamos ese ideal de la sociedad de la información y el conocimiento (SIC) (FUNIBER, 2020 pág. 51) mismo que es generador de los llamados educomunicadores o amateurs de los medios como Youtube, Facebook, Tik Tok que se convirtieron en los aliados perfecto del sistema educativo.

Mario Bunge trabajó este modelo de sociedad y señalaba en casi dos décadas antes del fenómeno de la pandemia que la sociedad de la información "(...) ha dado origen al mito de que el universo está compuesto de bits en lugar de materia". (Bunge, 2002, pág. 23) la llamada generación del big data da como resultado las transición de cambios generacionales en la que los dispositivos móviles son más comunes que un libro de historia. Lo anterior si bien nos pone en contexto no basta para detallar el papel de la enseñanza de esta asignatura en plena

pandemia, para ello haremos uso de algunas estadísticas que permiten la concepción desde los estudiantes hacia la clase.

Gracias a la investigación realizada, en la cual se tomó una sección de historia de Honduras, con una población de 143 jóvenes en edades que van desde los 17 a los 38 años; se realizaron una serie de interrogantes en encuestas semiestructurada que permitieron conocer las opiniones de la modalidad a distancia con sesiones interactivas cada quince días, el trabajo autónomo para cada estudiante, invitaciones de profesionales investigadores de áreas hermanas, espacios aleatorios de formación individual, una gama amplia de formación integral que generan auténticos espacios formativos multidisciplinarios.

Las encuestas recibieron la participación total de los jóvenes, dejando muy claro algunos elementos que son de pertinencia a los docentes del área en el nivel superior. Una de las primeras interrogantes era la condición en la que estas clases (historia, geografía, sociología, filosofía) eran realizables en el esquema en tiempo real, así como la importancia de la preparación teórica-informática del tutor académico, del mismo modo en que se consultó si la experiencia hasta entonces vivida era agradable o desagradable; cada una de las preguntas realizadas con una intencionalidad bien establecida, logra proyecciones que permitirá a otros docentes empoderarse.

Con base en la percepción de los jóvenes sobre la viabilidad de cursar clases interactivas, utilizando aplicaciones en tiempo real los resultados se muestran a continuación: 


\section{Gráfico 1}

Percepción de aceptación de las clases de ciencias sociales en modalidad interactiva (uso de aplicaciones en tiempo real)

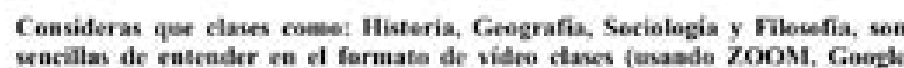
Mest, eatre atras)

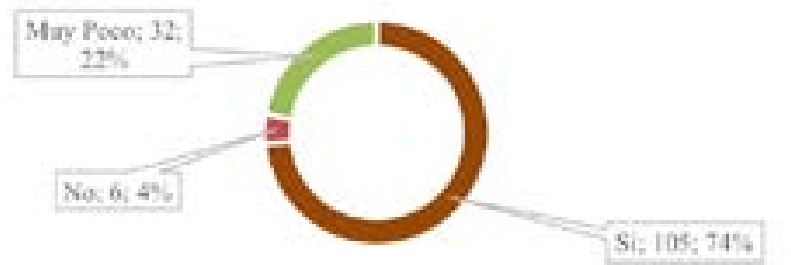

$-\mathrm{si}$

Nota: elaboración propia, con base en la tabulación de respuestas generadas a la encuesta aplicada, 2021

Este gráfico hace notar como la mayoría de los jóvenes han tenido experiencias amigables con las formas en que se transmiten los conocimientos. No es casualidad por algo que se les cite Multitasking, pues con cotidianidad reciben sus clases mientras realizan compras en los supermercados - con sus auriculares puestos- otros van de camino -conduciendo- y si algo no queda claro consultan en el momento y si persiste la duda consultan la grabación de la clase en los repositorios disponibles.

Añadiendo que muchos docentes han sido señalados por reusarse al cambio, algunos haciéndose virales en grabaciones donde se observan un retorno al siglo XIX escolástico, otra buena parte se ha actualizado convirtiéndose hasta en creadores de contenidos. En una de las interrogantes de estudio se analizó cómo consideraba la preparación de los docentes del área -señalando que algunos ya han tenido la experiencia con otros docentes, no necesariamente, el mismo para todas las clasesen sus diferentes espacios de aprendizajes.
Gráfico 2

Grados de evaluación a los docentes del área de ciencias sociales en esta nueva era de la educación (1 calificación mínima y 5 calificación máxima)

¿Cómo calificarias la preparación de los docentes al mononto de impartir cstas clases (Historia, Geografia, Sociologia y Filosofia)?

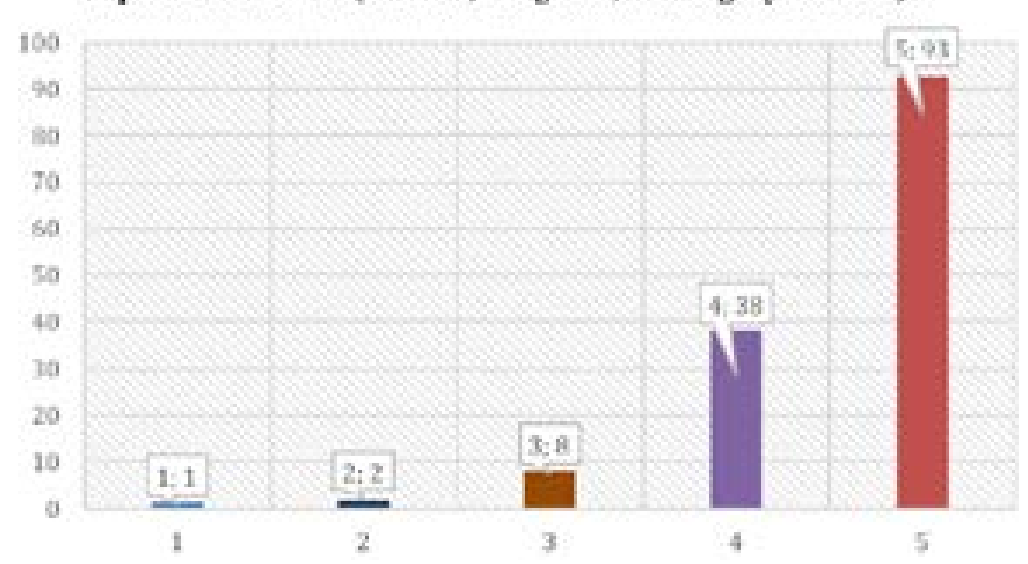

Nota: elaboración propia, con base en la tabulación de respuestas generadas en la encuesta aplicada, 2021.

Una de las nociones poco entendidas es que la educación sufrió la apertura de los espacios, es decir de aulas cerradas donde el docente era todólogo a espacios abierto, en donde los docentes del área de sociales saben que deben innovar, y apuntar a los portafolios de las experiencias hasta controlar el flipped classroom.

Esta condición de innovar por la necesidad de expandir la historia a espacios abiertos formativos nos da como respuesta que entre los mismos jóvenes se conversan con quien sí o no deben cursar la clase, no por la gran cantidad de memoria o el intelectualismo que posea el docente, sino por la sencillez y la capacidad informática de dar los contenidos.

Basándonos en la primicia del intercambio entre estudiantes, examinamos que tan viable es que recomienden entre ellos mismos cursar clases con esta modalidad, a lo que concluimos que si bien la mayoría apunta que sí lo recomienda, una cantidad considerable señala que no -algunos de ellos apuntaron a que el contacto físico con las clases es insuperable- 


\section{Gráfico 3}

Recomendación de cursar las clases modalidad interactiva, según los estudiantes consultados

\section{En tu experiencia como estudiante recomtendarias cursar estas clases et modalidad video clases (usando 7.00M, Google Meet, entre otros)}

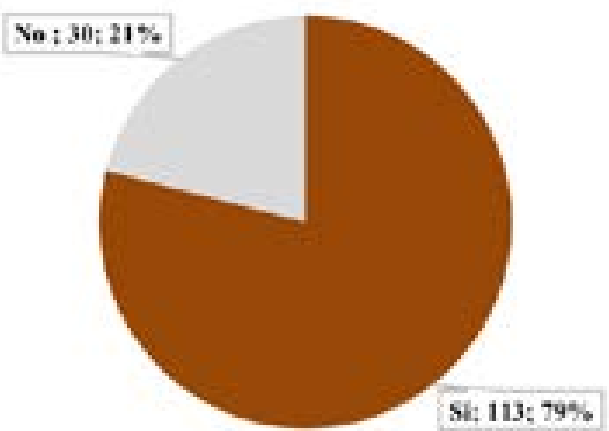

$\Delta \mathrm{Si}-\mathrm{Na}$

Nota: elaboración propia, con base en la tabulación de respuestas generadas a la encuesta aplicada, 202

La historia como ciencia, así como la enseñanza son procesos que no volverán a ser iguales, y en los cuales los profesores somos, sin miedo a equivocarme, los responsables de cómo crear la aceptación o bien la necesidad de que nuestras sociedades centroamericanas la busquen en su vida cotidiana. Esta responsabilidad no debe ser disminuida al contrario debe ser bienvenida, adecuadamente trabajada, ya que en este estudio de caso se concluye que la condición humana es lo más importante, apuntar de nuevo a la igualdad, la libertad, la solidaridad y la administración de las comunidades imaginadas. (Anderson, 2011) .

\section{Gráfico 4}

Nivel de importancia que posee en las clases de historia la condición humana del docente

Importancia de la condición humana en la clasc, es decir la energia con la que se transmiten los contenidos

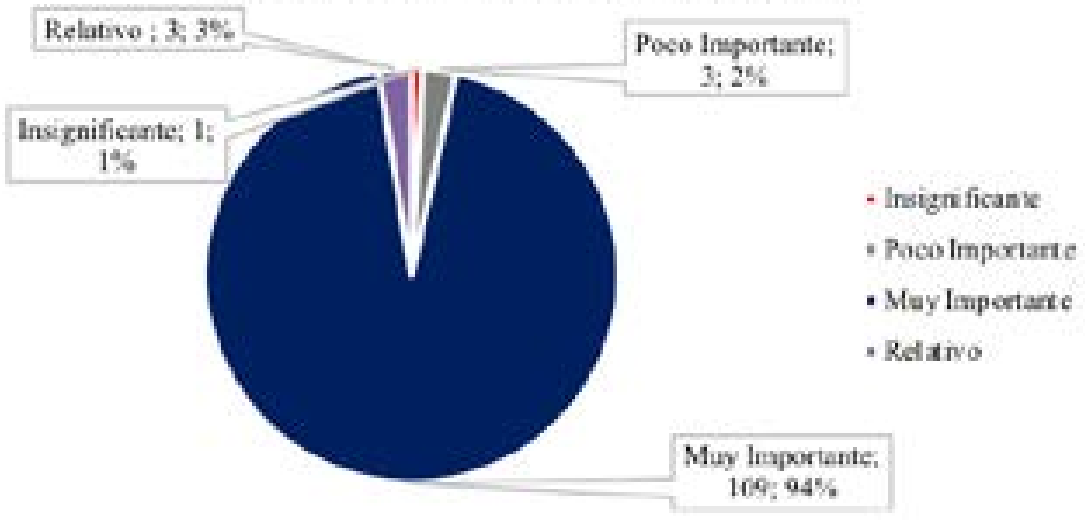

Nota: elaboración propia, con base en la tabulación de respuestas generadas a la encuesta aplicada, 202

En suma, las ciencias sociales, específicamente, la enseñanza de la historia en el nivel superior, se han logrado adaptar y reinventar, contando con la aceptación de los estudiantes, junto a una constante capacitación docente que permita la adecuada transmisión de los contenidos, sin embargo, este apartado solo da una visión general que será cerrada en el último apartado del artículo.

\subsection{La virtualización de las ciencias sociales en el nivel medio en Honduras}

La educación media, así como la prebásica y básica en el contexto hondureño han venido sufriendo una serie de adaptaciones desde la modificación casi total de las mallas o los programas curriculares para el año 2012-2013, sumada la emisión de una nueva ley fundamental de educación en Honduras. Procesos liderados por ingenieros agrónomos del país y no profesionales de la educación como en teoría debería ser.

A lo anterior, se le debe sumar que año con año el presupuesto para el sector educación y salud son los más afectados -en cuanto a 
disminución- a tal punto que el magisterio y la infraestructura de los centros educativos se hayan visto fuertemente deteriorados en la última década. (El Heraldo, 2019). Sin embargo, de una u otra forma las actualizaciones llegan a estos espacios formativos en su mayoría por injerencia directas de las llamadas organizaciones sin fines de lucro (ONG). haciendo una disminución en la responsabilidad estatal.

El año 2020 significó en Honduras la evidencia de las ineficiencias existentes y prolongadas por las mismas autoridades de esa secretaría de Estado, la falta de nombramiento, el secretismo en la manipulación de los presupuestos, la presión a los padres de familia para sustentar los centros educativos y el sacrificio del magisterio que por pasión al arte hace diferentes actividades para mantener la población estudiantil, se vio incrementada por la pandemia significativamente.

Según los estudios realizado por la Oficina Regional de Educación para América Latina y el Caribe de la Organización de las Naciones Unidas para la Educación, la Ciencia y la Cultura (OREALC/UNESCO) (CEPAL-UNESCO, 2020, pág. 1):

En el ámbito educativo, gran parte de las medidas que los países de la región han adoptado ante la crisis se relacionan con la suspensión de clases presenciales en todos los niveles, lo que ha dado origen a tres campos de acción principales: el despliegue de modalidad de aprendizaje a distancia, médiate utilización de una diversidad de formatos y plataformas (con o sin uso de tecnologías); el apoyo y movilización del personal y las comunidades educativas, y la atención a la salud y el bienestar integral de las y los estudiantes.

La verdad es que esta situación permitió cambiar la mirada sobre circunstancias de enorme importancia, como por ejemplo la necesidad de haber invertido más en educación prebásica, básica y media, capacitación docente y estructuras de conectividad. No maquillar tanto la realidad con proyectos que al final quedan olvidados, dirá el profesor de historia de las ideas morales: "acaso advirtamos que las desorbitadas inversiones en gastos militares no sirven para mucho (...) que resulta más rentable invertir en ciencia e innovación, cultura y educación, además de dotar al sistema sanitario público con los recursos apropiados" (Aramayo, 2020)

Al mismo tiempo que arreciaba la pandemia en el país algunos profesores comenzaron a intentar innovar desde las posibilidades de cada uno, encontrándose con el dilema ¿qué enseñar en historia?, ¿qué contenidos desarrollar en sociología?, ¿cómo enseñar en ciudadanía cívica? Bajo esa realidad casi ficticia es que se optó por un discurso resignificador de la ciencia, apuntando sobre todo al "rol social" y no como simples personas, sino como actores o sujetos inmersos en un fenómeno sociosanitario (CEPAL-UNESCO, 2020, pág. 11)

Las exigencias como lo señala Ricardo Sánchez no era pelear por un cursito o un espacio en la televisión para abarcar contenidos, es dejar huella para toda la existencia "la imagen que tenemos de otros pueblos, y hasta de nosotros mismos, está asociada a la historia tal como se nos contó cuando éramos niños" (Sánchez Cabanillas, 2020).

Uno de los discursos académicos más generalizados fue el de comprender el contexto de pandemia; analizar las respuestas sociales, los mecanismos de los gobiernos y las políticas que se estaban llevando a cabo, visualizar en comparación a otras situaciones cuando las democracias occidentales por ejemplo hacen uso de las suspensiones de garantías, retomar el concepto de "Estado" como garante de todos los ciudadanos y no solo un árbitro de intereses para capitales privados. (Franco, 2020)

Desglosar nuevamente, la necesidad de colectividad y no de individualización de las problemáticas, y si bien hubo un divorcio de los contenidos programados existió una alta concientización social en la que el niño en tiempos de clase, en notas de voz, en videos compartidos logró unificar su hogar haciendo que sus padres pudiesen escuchar, y dando como resultado un mayor impacto en las comunidades-aclarando que este último no se logró en todo el país-. Tras esas innovaciones cientos de docentes lograron autoprofesionalizarse en herramientas informáticas, así como en la distribución de materiales impresos para que los estudiantes lograrán avanzar, esta última con un mayor éxito a la primera, por la carencia misma de conectividad. Algunas aproximaciones señalan que de cada 10 hondureños solo 4 poseen Internet en zonas rurales y 7 en zonas urbanas (PNUD, 2020). 
En el caso específico nuestro el Instituto Oficial Villa Marco, de una totalidad de 108 estudiantes matriculados a través de la entrega de materiales impresos, se ha logrado estandarizar el trabajo para todos y cada uno de ellos; de parte oficial a través de la secretaria de educación se brindó materiales de trabajo para los jóvenes hasta el mes de septiembre del año 2020 (Secretaría de Educación de Honduras, 2020), lo cual como se señalizó anteriormente, no significo una limitante para los docentes, quienes ya poseían el trabajo avanzado. En el siguiente apartado daré una visión de cómo los profesionales en la enseñanza de la historia y ciencias sociales puede readecuar sus espacios formativos.

\section{Propuesta de la enseñanza de la historia en Honduras, de cara al Bicentenario de independencias}

Es crucial destacar que este apartado no tiene como finalidad establecer una fórmula normalizada para toda la región Centroamérica, sino realizar un intento, producto de las experiencias que se puedan compartir, los retos y sobre todo las dinámicas sociales que nos competen en este Bicentenario de las independencias centroamericanas, sumada la necesidad imprescindible de enseñar historia, construir conocimientos y realizar esos cambios de mentalidades urgentes en nuestra población.

Lo primero es definir en cuestión que la historia no es ninguna heterodoxia científica -no es fantástica- sino más bien un cúmulo de realidades producto de tiempos históricos definidos, de unidades da cambio, significaciones relativas, que variaran dependiendo de los sujetos, a la vez que cada causalidad de hechos o realidades no necesariamente, derivará en una situación optimista, en otras palabras, los cambios no siempre son efectivos a veces se falla y crean vulnerabilidades.

Víctor Hugo Acuña uno de los académicos del área más reconocidos en el nivel centroamericano, detallaba que la región "es producto de una historia y no de un destino", esto permite comprender esas continuidades, rupturas y discontinuidades, que al final darán a luz escenarios que vivimos, (Acuña, 2020).

\subsection{Contenidos o formación integral}

La amplia tarea que muchos docentes se han exigido es el cumplimiento de una malla curricular; su realización en sílabos o jornalizaciones de clases que intentan justificar el sueldo a como dé lugar, eso es uno de los mayores errores de los profesionales en la enseñanza de la historia. A doscientos años de independencia tanto en el nivel básico, medio y superior de educación memorizar no es una opción.

Un ejemplo que se puede comentar son las llamadas "Cortes de Cádiz" las cuales siempre son vistas como las generadoras de cambios que dan como resultado las independencias; la pregunta al dar este tema en lugar de enseñar diputado por diputado, reformas por reformas es ¿qué importancia concreta tiene esta en los estados actuales? -sin caer en el cortoplacismo- ¿cómo decirle a un joven de 16-20 años que debe comprender el proceso de transición monárquico al independiente?

Se podría perfectamente citar en este tema el caso de nuestras constituciones o emblemas nacionales que poseen leyendas como "LIBRE, SOBERANA E INDEPENDIENTE" ideales plasmados en el Artículo 2 de dicha constitución (Constitución de Cádiz 1812). Es primordial que más allá de crear enciclopedias andantes empecemos a generar ciudadanos comprometidos, sensibles a las situaciones actuales que nos incomodan y que con los conocimientos que facilitamos ellos puedan generar soluciones en sus vidas.

Esta situación de selección de contenidos no solo debe estar por una simple voluntad institucional de las universidades o los colegios, sino por la coherencia y la autonomía de cada docente. Aquí yo definiría la selección de contenidos como la comprensión humanista de temas relevantes que permitan siempre la concientización histórica, como necesidad de construcción y reconstrucción de esos significados.

Agregar que no es indispensable la totalización de todos los contenidos, pensar en la primicia que muchas veces menos es más y en lugar de continuar con el llenado de guías de trabajo podemos hacer reconstrucción de hechos con obras artísticas que pueden ir desde un dibujo, hasta una película u otro tipo de materiales. 


\subsection{Los estudiantes en la nueva enseñanza de la historia}

Si bien es cierto que la selección, la articulación y el desarrollo de contenidos es importante, el estudiante deberá desempeñar un papel más involucrado en esta nueva manera de aprender historia, la idea de "ir construyendo y enseñando a construir el conocimiento histórico". (Prats, 2001, pág. 66), somos responsables de hacer que esa conciencia histórica no sea simplemente de papel, sino que vaya encaminada a la generación de propuestas de cambio - dejar de predicar como si fuésemos del siglo XIX y ponernos por fin en el lugar temporal que tenemos que es el inicio del siglo XXI.

Los estudiantes que intenten aprender historia en los diferentes niveles, se les debe encaminar, que por ellos mismos sientan la necesidad de investigar su historia nacional, quizás, no a través de la típica historia política, sino complementando las nuevas corrientes que nos dan miradas poco conocidas de esos procesos que nos definen como "las patrias quebradas" en que nos hemos convertido.

Por ello, en este inciso propongo la instauración de investigaciones a acontecimientos plenamente conocidos en los imaginarios colectivos como la independencia de 1821 y luego de la presentación que realicen los estudiantes; llevar a cabo un feedback desde las nuevas perspectivas de la investigación como la prosopografía, el papel de las mujeres, la nueva historia política, la historia ambiental, historia social y cultural, que en cuestión permiten más que un hecho, conocer un contexto de la época.

Se deja ver que poco a poco en toda la estructura de este estudio se evidencia la relación entre la enseñanza y la investigación, esto porque nuestras sociedades están poco acostumbradas a que la teoría y la investigación histórica vayan de la mano, situación que no se puede permitir -al menos no en este estudio.
Figura 1

Esquema de como socializar el espacio pedagógico en la clase de Historia (siendo el estudiante el centro del proceso de enseñanza).

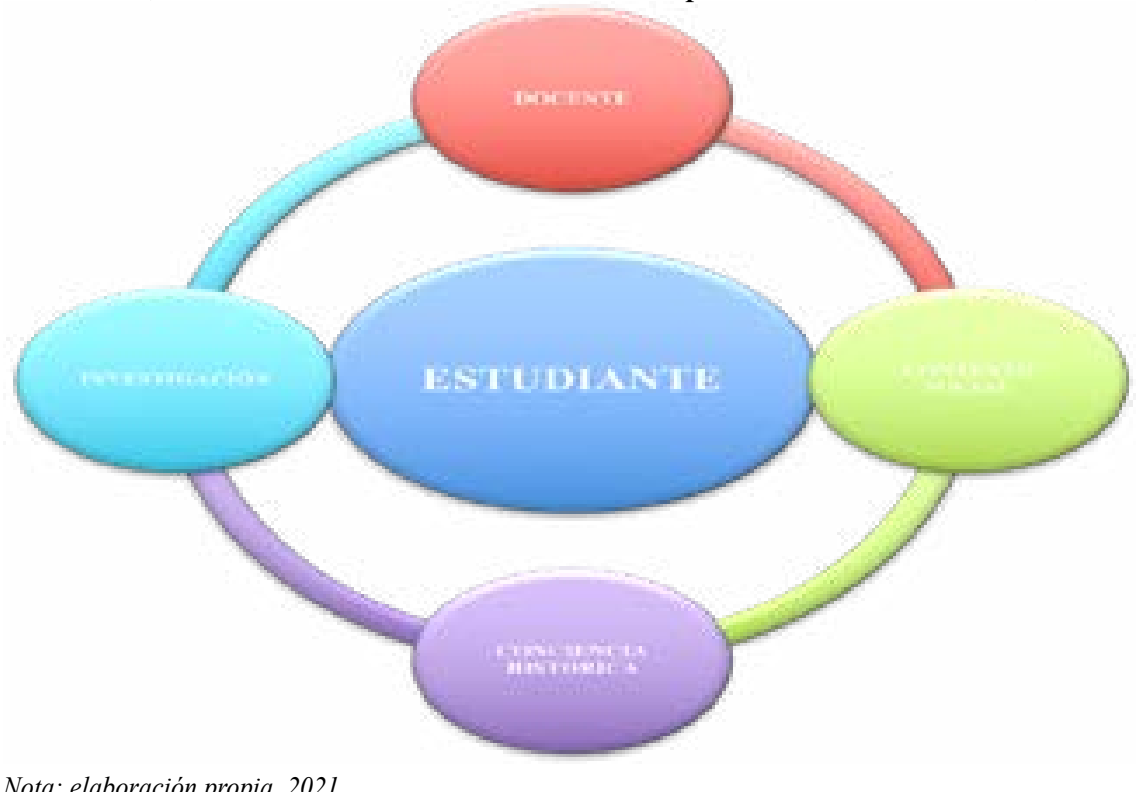

\subsection{Los docentes en la nueva enseñanza de la historia}

El profesional en la enseñanza de la historia se ha visto inmerso en el debate epistemológico de investigar o simplemente enseñar (Pagés Blanch, pág. 156), según mi experiencia estudiar para reproducir lecturas a una población estudiantil, no es suficiente; debe haber investigación para que las clases como tal manifiesten una comprensión adecuada.

Diré que el profesional en la enseñanza de la historia debe por obligación investigar, fundamentación teórico-metodológico de los contenidos. Esto no es ningún capricho a regaña dientes, sino que los jóvenes desde edades muy tempranas se ven en la necesidad de tomar decisiones, clasificación de la información -ignorar mucho, para aprender poco- y eso solo puede lograrse a través de los métodos históricos.

En el estudio de caso los estudiantes señalaban que uno de los elementos más importantes de la educación es la energía -dirán los jóvenes 
de hoy en día: las buenas vibras- con que el docente comparte los saberes; una visión muy compleja, pues debemos recordar que en esta época desempeñamos el papel de coaching de la educación, en otras palabras, nos hemos convertido en motivadores hacia los estudiantes con el propósito de mantener un nivel de retención estudiantil elevado.

Este inciso intenta esclarecer el papel diferente del docente, clave de la mediación pedagógica que intenta siempre la construcción del aprendizaje significativo. En historia han existido estereotipos de como son los profesores que enseñan esta disciplina; aquel que narra las historias, el profesor científico, el filósofo cósmico, historiador ecléctico y el más común en Honduras el reformista contra sistema (Pagés Blanch, págs. 59-69)

Estas concepciones reales en mayor o menor medida son claves para que un estudiante pueda sentirse atraído a los contenidos, pero, ¿cuál es el problema en el caso de Honduras? que los profesores que enseñan historia no son del área, es más ni siquiera afines al área de las ciencias sociales, esto marca una gran desventaja en comparación a espacios como los europeos o los mexicanos en que los profesores se encuentran planamente especializados.

Es aquí donde la ética del profesional se ve truncada, sin embargo, más allá de esa situación el papel de los profesores de historia y ciencias sociales es el de dar lección para la toma de decisiones en un marco de causalidades, poner en práctica los conceptos de "análisis", "descripción" e "interpretación". Estos tres conceptos nos permitirán comprender la complejidad histórica, invisibilizada en pleno Bicentenario centroamericano, donde las matemáticas, la biología, la química, el español y el inglés son las únicas clases con nivel elemental para nuestros sistemas educativos. La verdad es que cada docente del área debe asimilar lo más rápido posible que debemos acercarnos a los contextos sociales de nuestros estudiantes, comprender la mentalidad de ellos -no juzgarla- y comenzar a crear una necesidad por el aprendizaje. Pensemos como si fuésemos estudiante ¿qué quiero realmente saber?, ¿cuál es la utilidad de este contenido?, ¿de qué me sirve aprender esto?

Hasta qué punto puedo yo apasionar a mis estudiantes para el aprendizaje de una historia propositiva que busca soluciones, que entiende los procesos y que busca ser concebida no como llave del

futuro, sino como esencia del escenario actual vivido. Son estos retos los que nos engloban, provocar hambre de historia en lugar de exigir memorizarla, comprometer a las comunidades estudiantiles de los diferentes niveles en contraposición a salir del paso -como se dice al finalizar el examen de curso o cursar la asignatura.

La historia es elemental en las vidas de nuestras naciones, es la única substancia que nos permite recomponer hechos, revivir experiencias que se vuelven difusas en situaciones como la pandemia por coronavirus, la historia es por sí misma la retrospectiva de una multitud de fenómenos que debemos volver a estudiar, con el propósito de conocernos y eso solo lo lograrán docentes profesionales de la historia, comprometidos con las sociedades en las que viven y aquellas en la que sueñan vivir.

\section{Hallazgos}

La riqueza de la investigación queda evidenciada en este estudio de caso, por lo cual no se busca ser un recetario de tareas por hacer o no, sino más bien poner en cuestión la necesidad de conocer las condiciones en los cuales se encuentran ejerciendo la función docente. Ese saber científico que solo la historia nos permite crear-investigaciones- es la mayor herramienta que podemos utilizar, ya que una vez adoptada la experiencia se puede compartir saberes que orienten a nuestros jóvenes a humanizar más la sociedad, a comprender las complejidades humanas a través del tiempo y ser sensatos en el papel que desempeñan durante los procesos de vida.

Una de las circunstancias que más cuesta a los historiadores investigadores y profesores- es la ubicación temporal, no es lo mismo hablar de tiempo en las ciencias sociales que en la física, debemos centrarnos en Longue durée ver la superestructura de las sociedades como la centroamericana, sus unidades de cambio y realidades. Aceptar que las complejidades históricas en lugar de ser una limitante representan múltiples posibilidades de enseñanza, no es mejor ser marxista, creerse posmoderno o sentir afinidad por la escuela de los Annales. Hay que trasladar esa revolución historiográfica al plano de la enseñanza. "Los

Perspectivas, cambios y resignificación de la enseñanza de la historia en Honduras del Bicentenario 
problemas de la enseñanza de la historia debe ser propuestas y resultas por los historiadores” (Sánchez Quintar, 1995, pág. 4)

La nueva visión o la visión que se debe adoptar en la enseñanza es la de "capital científico" los descubrimientos de investigaciones que dan nuevas comprensiones a la problemática que se viva -hacer ver a la sociedad que no todo es producto de alienígenas ancestrales- que hay todo un cimiento en la reconstrucción de un hecho histórico. (Bordieu, 1994). Pero lo más importante, si esta ciencia tiene legitimidad en la sociedad actual es solo porque desde la historia se puede realizar una mejor aproximación a los hechos que muchas veces son arrebatados por otros saberes, ejemplo de lo antes mencionado es la funcionalidad de los vínculos sociales, la reconstrucción de nuestras identidades y del sentido de ciudadanía, la constante lucha de reflexionar los acontecimientos para dar sentido a la realidad actual, las convivencias humanas y sobremodo dar protagonismo a los actores colectivos. Historia es para Honduras la posibilidad de rescatar lo que hasta este momento representa para millones de ciudadanos un Estado fallido, por ello, es la invitación abierta a la creación de diálogos que permitan el intercambio de ideas, experiencias o propuestas que nos orienten a una sociedad mejor.

\section{Bibliografía}

Acuña, V. H. (30 de 9 de 2020). Centroamérica en la larga duración: 2020-1821. Virtual, Costa Rica: Universidad de Costa Rica.

Anderson, B. (2011). Comunidades imaginadas: reflexiones sobre el origen y la difusión del nacionalismo. México: Fondo de Cultura Económica.

Aramayo, R. (22 de marzo de 2020). THE CONVERSATION. Obtenido de https:// theconversation.com/reflexiones-desde-la-filosofia-lo-que-covid-19-puede-ensenarnos-134023

Bejerano, P. G. (6 de febrero de 2014). elDiario.es. Recuperado el 11 de 3 de 2021 , de https://www.eldiario.es/turing/criptografia/alan-turing-enigma-codigo_1_5038272.html

Bordieu, P. (1994). El campo científico. Redes: Revista de Estudios Sociales de la Ciencia, 129-160. Recuperado el 10 de febrero de 2017, de https://ridaa.unq edu.ar/handle/20.500.11807/317

Bunge, M. (2002). Crisis y reconstrucción de la filosofía . Barcelona: Gedesia S. A.
CEPAL-UNESCO (2020). Informe COVID-19 La Educación en tiempos de la pandemia de COVID-19. Oficina Regional de Educación para América Latina y el Caribe de la Organización de las Naciones Unidas. CEPAL-UNESCO.

Constitución de Cádiz 1812 (S. F.). Obtenido de http://www.diputados.gob.mx/biblioteca/bibdig/const_mex/const_cadiz.pdf

Diario La Prensa (27 de enero de 2020). Las universidades buscan reducir la brecha digital. Diario La Prensa. Recuperado el 11 de marzo de 2021, de https://www.laprensa.hn/economia/dineroynegocios/1352236-410/ universidades-buscan-reducir-brecha-digital-honduras

El Heraldo (27 de mayo de 2019). Siete datos para entender el conflicto educación y salud en Honduras. Recuperado el 11 de marzo de 2021, de https://www. elheraldo.hn/pais/1288002-466/siete-datos-para-entender-el-conflicto-en-salud-y-educaci\%C3\%B3n-en-honduras

Franco, M. (28 de marzo de 2020). PERFIL. Recuperado el 10 de febrero de 2021 , de https://www.perfil.com/noticias/universidades/el-estudio-de-la-historia-en-tiempos-de-pandemia.phtml

Fujuyama, F. (S. F.). Alianza Editorial. Recuperado el 11 de marzo de 2021, de https:// www.alianzaeditorial.es/minisites/manual_web/3491295/CAP8/1_FindelaHistoria.pdf

FUNIBER (2020). Entornos comunicativos y aprendizaje en la SIC. En Sociedad de la información y el cambio (págs. 51-102). España: FUNIBER.

Guldi, J., \& Armitage, D. (2016). Manifiesto por la historia. Madrid, España: Alianza Editorial.

Pagés Blanch, J. (S. F.). Enseñar a enseñar Historia: La formación didáctica de los futuros profesores de historia. Miradas a la historia, 155-178.

Pantoja Suárez, T. (2017). Enseñar Historia, un reto entre la didactica y la disciplina: reflexión desde la formación de docentes de Ciencias Sociales en Colombia Diálogo Andino(53), 59-71.

Parrales, M. (14 de 5 de 2020). El profesorado y la educación virtual: experiencias en la región centroamericana. Espacio Virtual, Costa Rica: Universidad Nacional de Costa Rica-Proyecto Educación Continua para Docentes de Estudios Sociales y Cívica.

PNUD (4 de octubre de 2020). Internet y juventud en Honduras. Recuperado el octubre de 2020, de https://scontent.ftgu2-2.fna.fbcdn.net/v/ t1.0-9/120816616_3361674533869402_1800827290410735360_o png?_nc_cat $=106 \& c c b=1-3 \& \_n c \_s i d=730 e 14 \& \_n c \_e u i 2=A e E u F h U V$ se HiNR-JWDRMagZOu6ysZ9IuytC7rKxn0i7K0E6Z2-3yfup4FtKNGJ-E-IMCB5gqrC3d9rawpefACn9p\&_nc_ohc=fhBR0_JuupIAX 
Prats, J. (2001). Enseñar Historia: notas para una didáctica renovadora. Merida: Junta de Extremadura Consejería de Educación, Ciencia y Tecnología.

Sánchez Cabanillas, R. (2020, agosto 21). Noticierolibre.com. Retrieved febrro 10, 2021, from https://noticierolibre.com/210820/ la-ensenanza-de-la-historia-en-tiempos-de-pandemia/

Sánchez Quintar, A. (abril-junio de 1995). Enseñar Historia en la universidad y fuera de ella. Perfiles Educativios(68), 1-7. Recuperado el 15 de febrero de 2017, de https://www.redalyc.org/articulo.oa?id=13206809

Secretaría de Educación de Honduras (25 de septiembre de 2020). Obtenido de https:// www.se.gob.hn/detalle-articulo/1488/

UNAH (13 de marzo de 2020). Estratégica, Blog Dirección de Comunicación. Recuperado el 11 de marzo de 2021, de https://blogs.unah.edu.hn/dircom/ consejo-de-educacion-superior-recomienda-suspender-actividades-academicas-por-21-dias 